\title{
Ego-Motion Estimation using Distributed Single-Channel Radar Sensors
}

\author{
Maximilian Steiner, Omar Hammouda, and Christian Waldschmidt
}

(C) 2018 IEEE. Personal use of this material is permitted. Permission from IEEE must be obtained for all other uses, in any current or future media, including reprinting/republishing this material for advertising or promotional purposes, creating new collective works, for resale or redistribution to servers or lists, or reuse of any copyrighted component of this work in other works. 


\title{
Ego-Motion Estimation using Distributed Single-Channel Radar Sensors
}

\author{
Maximilian Steiner, Omar Hammouda, Christian Waldschmidt \\ Institute of Microwave Engineering \\ University Ulm \\ 89081 Ulm, Germany \\ Email: Maximilian.Steiner@uni-ulm.de
}

\begin{abstract}
The motion of a car has a vast impact on the relative measurements of all environmental sensors. Consequently, the precise knowledge of the ego-motion of a sensor equipped car is important for higher level signal processing like mapping or the estimation of the target parameters. This can be achieved by multiple simple radar sensors with a single measurement. The range and the velocity measurements conducted by multiple sensors that are observing a common field of view are jointly processed and filtered for stationary targets. This dataset is used to determine the velocity and the yaw rate of the vehicle in the $x$ - and $y$-plane. An advantage of the proposed approach is the simplicity of the sensors which do not need the capability of angle estimation in the azimuth plane, while still providing comparable results. In addition, this approach works with a minimum number of two sensors.
\end{abstract}

\section{INTRODUCTION}

The knowledge of the actual ego-motion of a car is an indispensable presupposition for future advanced driver assistance systems as well as for automated driving. In particular, applications that depend on multiple measurements of an environmental sensor (mm-wave or optical) during the movement or deal with moving targets require the knowledge of the ego-motion. Examples of such applications are grid-mapping, target-tracking, and estimation of the actual target movement (e.g. direction and velocity). Recent trends show a growing number of radar sensors surrounding a car for various tasks. Due to the rapid progress in the integration of radar sensors, cost efficient simple sensors are feasible. For the approach discussed in this publication, radar sensors with only one receive channel are sufficient what allows a very small form factor. This simplifies the placement on a car contour and increases the robustness of the system with respect to sensor failures. In comparison to state-of-the-art radar sensors which utilize angle of arrival estimation techniques to determine the position of a target, the information of the simple sensors is reduced to the range-Doppler domain. The measurements of all sensors with a common field of view are triggered at the same time. After extraction of the range and velocity information of all targets, a joint processing results in the actual motion parameters.

\section{Sensor Setup and Main Equation}

The sensors are distributed around the vehicle with at least two sensors on each side to be able to calculate the angles at which the targets are located as shown in Fig. 1. These angles are needed to relate the radial velocities measured from the targets to the full 2D-motion of the car.

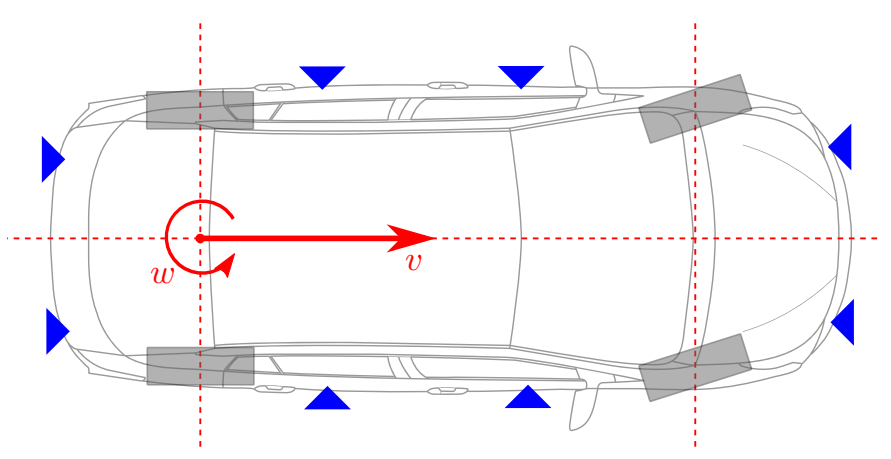

Fig. 1. Exemplary distribution of sensors around the car [1].

In a linear motion case where the car is not exhibiting any rotations, all points on the car contour have the same velocity profile. However, as the car starts rotating, different points on the car have distinct velocity profiles depending on the distance to the pivot of rotation. Therefore, the full 2D-motion of the car is described by the movement of the rear axle midpoint, where $v$ represents the linear motion of the car in $\mathrm{m} / \mathrm{s}$ and $\omega$ represents the car yaw rate in $\mathrm{deg} / \mathrm{s}$.

The main equation relating the measured radial velocity from a stationary target to the full 2D-motion of the car is

$$
\begin{aligned}
-V_{j, i}^{r}= & \left(-y_{j}^{s} \cos \left(\theta_{j, i}\right)+x_{j}^{s} \sin \left(\theta_{j, i}\right)\right) w \\
& +\cos \left(\theta_{j, i}\right) v_{x}+\sin \left(\theta_{j, i}\right) v_{y},
\end{aligned}
$$

where $i=1,2, \ldots, N_{j}$ refers to a certain target per sensor and $j=1,2, \ldots, M$ points to the sensor number [2]. The sensor positions are described by $\left(x_{s}, y_{s}\right)$. The measured radial velocity and the calculated azimuth angle are denoted by $V_{j, i}^{r}$ and $\theta_{j, i}$, respectively. It can be seen from (1) that the mounting location of the sensor must be accurately determined to correctly calculate the angles at which the targets are located and also to correctly determine the value of $\omega$ if a rotation is exhibited. If the target is moving, the measured radial velocity cannot be used to calculate the ego-motion as it does not only represent the motion of the sensors but also the target movement. 


\section{Ego-Motion Estimation}

The flowchart shown in Fig. 2 gives an overview about the algorithm used for ego-motion estimation. Each step is detailed in the following subsections.

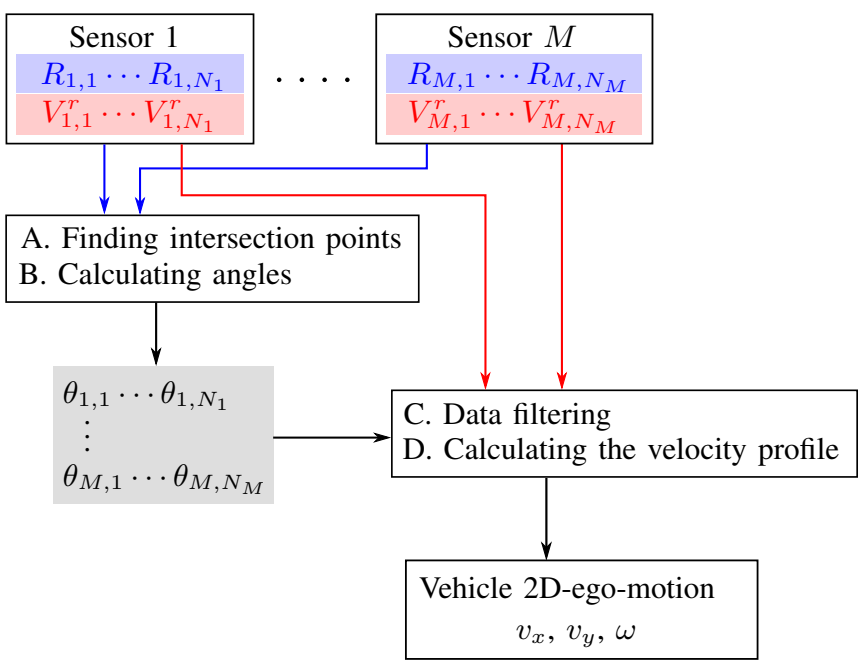

Fig. 2. Algorithm overview.

\section{A. Finding Intersection Points}

The first step in the algorithm has to be the calculation of the angle at which a target is located. This angle information that is needed to estimate the ego-motion is not provided by a single radar sensor. This is done by first finding the target locations through a bilateration process, in which range data is used to find intersection points. If the range measurements taken from two different sensors belong to the same target, the resulting target intersection point correctly represents a real position. However, if the range data used in the bilateration process belong to two different targets, the resulting intersection point does not represent any real target. This intersection point is referred to as a false point. After finding an intersection point, the angle between this point and the sensor is calculated, and the corresponding measured radial velocity is assigned.

\section{B. Calculating Angles}

Since two sensors are involved in finding an intersection point, each intersection point gives two sets of data. Each data set includes the sensor location, the angle between the intersection point and the sensor, and the radial velocity corresponding to the range information used in finding the intersection point. By inserting this data set in (1), the only unknowns remaining are $\omega, v_{x}, v_{y}$, which represent the velocity profile of the car. At least three equations are needed to be able to estimate the full 2D-motion of the car.

As mentioned before, not all equations will result in estimating the correct velocity profile. This is due to false points which have incorrect angles as they do not point to real targets in the scene and due to intersection points related to moving

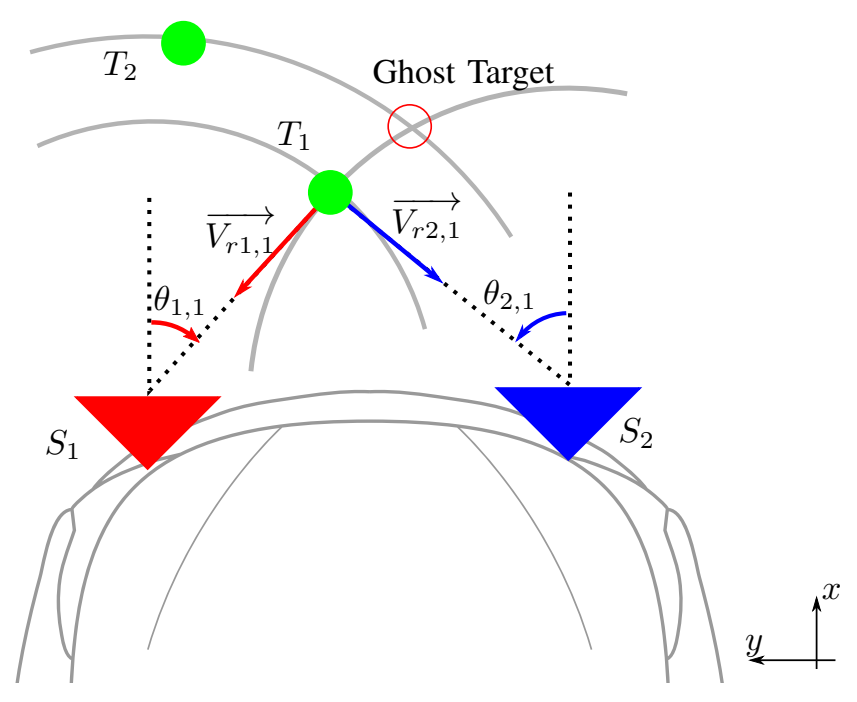

Fig. 3. Depiction of the velocity behavior of a target with respect to each sensor and a ghost target $(\mathrm{O})$ due to the intersection between range circles belonging to two different targets (๑).

targets as their measured radial velocities do not represent the cars motion. For this reason, data filtering needs to take place before the ego-motion can be estimated. Here, this is done by using the RANdom SAmple Consensus (RANSAC) algorithm [3]. This algorithm has the advantage of extracting the largest number of points that follow a linear model without the need of any information about the number of valid points and their behaviors. Therefore, in any scene an assumption is made, that the points belonging to stationary targets are the largest number of points which have the same behaviors.

\section{Data Filtering}

To emphasize the importance of the data filtering on the estimated ego-motion, a simulation is done where a car is moving with a forward speed of $10 \mathrm{~m} / \mathrm{s}$. By directly calculating the velocity profile parameters given all data points, the result is $\omega=-44.3 \mathrm{deg} / \mathrm{s}, v_{x}=-7.91 \mathrm{~m} / \mathrm{s}, v_{y}=-0.22 \mathrm{~m} / \mathrm{s}$. As observed, the result is far from the ground truth with a significant margin, rendering this method to estimate the egomotion useless. By applying the RANSAC algorithm, the egomotion is calculated based on only the inlier points, which are shown in Fig. 4. This results in the estimated velocity profile being equal to $\omega=1.3 \mathrm{deg} / \mathrm{s}, v_{x}=10.02 \mathrm{~m} / \mathrm{s}, v_{y}=0.006 \mathrm{~m} / \mathrm{s}$, which is very close to the ground truth. This proves that the RANSAC algorithm is necessary for a correct estimation of the model parameters within an acceptable margin as it removes intersection points of moving and false points that affect the estimation of the ego-motion of the car. The algorithm is used to find the best fit for a set of data with outliers. It is based on two main steps. First, the outliers are removed, and afterwards the best fit is calculated only for the inliers. The algorithm iterates taking the minimum number of data points required to calculate the model parameters. Then it checks the number of inliers based on the proposed model. A data point is counted 


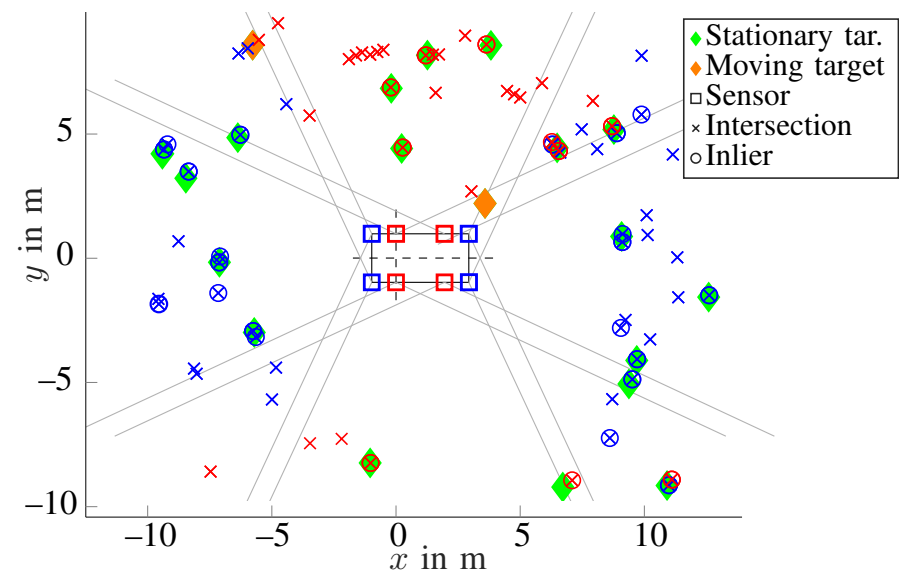

Fig. 4. Top view of the simulation of a moving vehicle with targets in the scene and the calculated intersection points. The chosen inlier points in the different observation areas are shown after applying the RANSAC algorithm.

as an inlier if it falls within a specified threshold from the model. To understand how the RANSAC algorithm works and why some set of points were chosen as inliers, the behavior of the data points needs to be observed. Some data points follow the ground truth, within a certain error threshold, while others have entirely different behaviors. The task is now to extract the data points that follow the ground truth without having any prior knowledge about the ground truth behavior. RANSAC does that by first randomly choosing the minimum number of points needed to build a model, then the number of data points that agree with this model, within a certain error threshold, are counted. The error threshold $\epsilon$ must be chosen large enough to accommodate the error in the inlier data points, but if chosen too large some points that not represent the model are considered and will result in an incorrect best-fit calculation. This process is repeated $N$ times, where in each iteration a new set of points is chosen and again a model is built and the number of points agreeing with this model are counted. In the end, the largest number of data points that agree with the same model are chosen.

To ensure the success of the algorithm, the number of iterations $N$ should be chosen high enough to guarantee that with a probability $p$ at least one of the random sets chosen does not include an outlier. If the probability of selecting an inlier in the data set is $u$ and the number of data points chosen in each iteration is $m$, with

$$
p=1-\left(1-u^{m}\right)^{N},
$$

the minimum number of iteration $N$ to ensure success with a probability $p$ can be calculated as

$$
N=\frac{\log (1-p)}{\log \left(1-u^{m}\right)} .
$$

\section{Calculating the Velocity Profile}

After filtering the dataset, the car ego-motion is calculated based on only the inliers chosen by the RANSAC algorithm.
The model parameters are fit using the Least SQuare (LSQ) estimation. The LSQ regression is optimum to use for the application discussed in this paper as the dataset is usually much larger than the number of model parameters [4]. Although there are better algorithms that give more accurate results, the LSQ still performs very well with a much lower complexity, making it suitable for real time applications. The main disadvantage of LSQ regression is its sensitivity to outliers, however, this is not a problem in the presented algorithm as the RANSAC applied in the previous step ensures the removal of all outliers.

The function of the data that can be fit using a LSQ regression should have the form

$$
f(\vec{x}, \vec{\beta})=\beta_{0}+\beta_{1} x_{1}+\beta_{2} x_{2}+\ldots .
$$

By comparing (4) to (1), it is observed that the model parameters are $\beta_{0}=0, \beta_{1}=\omega, \beta_{2}=v_{x}, \beta_{3}=v_{y}$, and the independent variables $x_{i}$ are $x_{1}=-y_{j}^{s} \cos \left(\theta_{j, i}\right)+x_{j}^{s} \sin \left(\theta_{j, i}\right), x_{2}=\cos \left(\theta_{j, i}\right)$, $x_{3}=\sin \left(\theta_{j, i}\right)$, and finally the measured variable is $-V_{j, i}^{r}$.

The system of equations for one sensor in matrix form can be expressed as

$$
\underbrace{\left[\begin{array}{c}
-V_{j, 1}^{r} \\
-V_{j, 2}^{r} \\
\vdots \\
-V_{j, N_{j}}^{r}
\end{array}\right]}_{\mathbf{V}_{\mathbf{j}}^{\mathbf{r}}}=\underbrace{\left[\begin{array}{cc}
\cos \left(\theta_{j, 1}\right) & \sin \left(\theta_{j, 1}\right) \\
\cos \left(\theta_{j, 2}\right) & \sin \left(\theta_{j, 2}\right) \\
\vdots & \vdots \\
\cos \left(\theta_{j, N_{j}}\right) & \sin \left(\theta_{j, N_{j}}\right)
\end{array}\right]}_{\mathbf{M}_{\mathbf{j}}} \cdot \underbrace{\left[\begin{array}{ccc}
-y_{j}^{s} & 1 & 0 \\
x_{j}^{s} & 0 & 1
\end{array}\right]}_{\mathbf{S}_{\mathbf{j}}} \cdot\left[\begin{array}{c}
w \\
v_{x} \\
v_{y}
\end{array}\right]
$$

Combining all $M$ sensors, the system of equations relating the measured radial velocities of targets $\mathbf{V}^{\mathbf{r}}$ from all sensors to the velocity profile parameters $\mathbf{V p}$ at the reference point is expressed as

$$
\underbrace{\left[\begin{array}{c}
\mathbf{V}^{\mathbf{r}}{ }_{1} \\
\mathbf{V}^{\mathbf{r}}{ }_{2} \\
\vdots \\
\mathbf{V}^{\mathbf{r}}
\end{array}\right]}_{\mathbf{V}^{\mathbf{r}}}=\left[\begin{array}{c}
\mathbf{M}_{1} \cdot \mathbf{S}_{1} \\
\mathbf{M}_{2} \cdot \mathbf{S}_{2} \\
\vdots \\
\mathbf{M}_{\mathbf{M}} \cdot \mathbf{S}_{\mathbf{M}}
\end{array}\right] \cdot \underbrace{\left[\begin{array}{c}
w \\
v_{x} \\
v_{y}
\end{array}\right]}_{\mathbf{V}}
$$

Calculating a model fit with the LSQ assumes that the error is only contained in the measured variable, and thus calculates a fit that minimizes the error with respect to the measured variable. This is however not true in the presented case, as the variable $\theta_{j, i}$ is also calculated from the measured target ranges which contain errors. Here, the Orthogonal Distance Regression (ODR) estimator might lead to better results at higher computation time.

\section{Simulations Results}

The performance of the algorithm is tested by simulating a car once with two sensors on each side separated by a distance of 2 meters, and again by three sensors on each side separated by a distance of 1 meter. The effect of two parameters on the performance is tested. First, the bandwidth is varied from $1 \mathrm{GHz}$ to $2 \mathrm{GHz}$ while the observation time is kept at $12.8 \mathrm{~ms}$, 
then the observation time is varied from $0.8 \mathrm{~ms}$ to $25.6 \mathrm{~ms}$ while the bandwidth is kept at $2 \mathrm{GHz}$. In both simulations two different velocity profiles are tested. In case $\mathrm{A}$, the simulation is run with $v_{x}=10 \mathrm{~m} / \mathrm{s}, v_{y}=0 \mathrm{~m} / \mathrm{s}$ and $\omega=0 \mathrm{deg} / \mathrm{s}$. In case $\mathrm{B}$, the simulation is carried out with $v_{x}=10 \mathrm{~m} / \mathrm{s}, v_{y}=0.1 \mathrm{~m} / \mathrm{s}$ and $\omega=5 \mathrm{deg} / \mathrm{s}$. The results of both tests are presented in the Tables I and II in terms of the Root Mean Square Error (RMSE) of $v_{x}$ and $\omega$. As expected the performance for both sensor settings

TABLE I

SIMULATION RESULTS IN TERMS OF RMSE AFTER TESTING THE EFFECT OF DIFFERENT OBSERVATION TIMES, WHERE $v_{x}$ IS MEASURED IN $\mathrm{m} / \mathrm{s}$ AND $\omega$ IN $\mathrm{deg} / \mathrm{s}$. DIFFERENT SCENARIOS ARE TESTED, WHERE A,B REFER TO THE MOTION BEHAVIOR AND 2,3 REFER TO THE NUMBER OF SENSORS PER SIDE.

\begin{tabular}{ll|cccccc} 
& & \multicolumn{6}{|c}{ Observation time $(\mathrm{ms})$} \\
& & 0.8 & 1.6 & 3.2 & 6.4 & 12.8 & 25.6 \\
\hline \multirow{2}{*}{$\mathrm{A}, 2$} & $v_{x, \text { rmse }}$ & 0.990 & 0.653 & 0.233 & 0.122 & 0.049 & 0.040 \\
& $\omega_{\text {rmse }}$ & 13.35 & 9.515 & 4.919 & 2.582 & 1.321 & 1.314 \\
$\mathrm{~A}, 3$ & $v_{x, \text { rmse }}$ & 0.816 & 0.508 & 0.163 & 0.071 & 0.050 & 0.041 \\
& $\omega_{\text {rmse }}$ & 10.66 & 6.966 & 3.485 & 2.087 & 1.683 & 1.602 \\
$\mathrm{~B}, 2$ & $v_{x, \text { rmse }}$ & 0.982 & 0.660 & 0.229 & 0.128 & 0.049 & 0.040 \\
& $\omega_{\text {rmse }}$ & 13.26 & 9.417 & 4.920 & 2.650 & 1.343 & 1.322 \\
$\mathrm{~B}, 3$ & $v_{x, \text { rmse }}$ & 0.820 & 0.522 & 0.160 & 0.072 & 0.050 & 0.042 \\
& $\omega_{\text {rmse }}$ & 10.89 & 7.001 & 3.515 & 2.113 & 1.715 & 1.547
\end{tabular}

TABLE II

TESTING THE EFFECT OF CHANGING THE BANDWIDTH.

\begin{tabular}{ll|ccccc} 
& & \multicolumn{5}{|c}{ Bandwidth $(\mathrm{GHz})$} \\
& & 1.00 & 1.25 & 1.50 & 1.75 & 2.00 \\
\hline $\mathrm{A}, 2$ & $v_{x, \text { rmse }}$ & 0.128 & 0.077 & 0.062 & 0.055 & 0.049 \\
& $\omega_{\text {rmse }}$ & 3.036 & 2.463 & 1.872 & 1.636 & 1.395 \\
$\mathrm{~A}, 3$ & $v_{x, \text { rmse }}$ & 0.163 & 0.078 & 0.066 & 0.055 & 0.048 \\
& $\omega_{\text {rmse }}$ & 3.422 & 2.883 & 2.465 & 2.141 & 1.752 \\
$\mathrm{~B}, 2$ & $v_{x, \text { rmse }}$ & 0.134 & 0.081 & 0.065 & 0.056 & 0.050 \\
& $\omega_{\text {rmse }}$ & 3.045 & 2.319 & 1.924 & 1.655 & 1.351 \\
$\mathrm{~B}, 3$ & $v_{x, \text { rmse }}$ & 0.179 & 0.078 & 0.064 & 0.059 & 0.051 \\
& $\omega_{\text {rmse }}$ & 3.609 & 2.985 & 2.443 & 2.059 & 1.784
\end{tabular}

with different vehicle motions improves significantly with the increase in the observation time and bandwidth. This is due to the fact that by increasing the observation time, the radial velocity resolution is improved. Similarly, by increasing the bandwidth the range resolution is improved. This simulation does not regard the fact that an increasing observation time might lead to a slightly worse performance due to range cell migration errors than what is shown in Table I. A second observation is that an addition of a rotation and a side slip have a negligible influence on the results. The reason for this is the estimator used in the algorithm, that has 3 Degrees Of Freedom (DOF).

\section{COMPARISON TO MEASUREMENTS}

The function of the algorithm is tested with measurement data that was captured during a linear motion.

The measurement is conducted utilizing three $77 \mathrm{GHz}$ radar sensors that are mounted at a height over ground of $60 \mathrm{~cm}$ on the right side of a car. The distance between the middle sensor and the rear sensor is $0.87 \mathrm{~m}$, while the front sensor is detached by $1.87 \mathrm{~m}$. The sensors are operated with a chirpsequence modulation covering a bandwidth of $1 \mathrm{GHz}$ and
$24.3 \mathrm{~ms}$ observation time. All sensors are pointing to the side, while the car is moving linearly with a velocity of $5.03 \mathrm{~m} / \mathrm{s}$, estimated by a GNSS+IMU system. Overall 10 radar targets are distributed over an area of $4 \mathrm{~m}$ times $6 \mathrm{~m}$. All targets that are detected by a OS-CFAR algorithm are processed. The estimation of 2 DOF results in $v_{x}=4.956 \mathrm{~m} / \mathrm{s}$ and $v_{y}=-0.021 \mathrm{~m} / \mathrm{s}$, while the 3 DOF estimation measurement results in a yaw-rate of $0.1 \mathrm{deg} / \mathrm{s}$, a velocity in $x$-direction of $4.872 \mathrm{~m} / \mathrm{s}$ and a velocity in $y$-direction of $-0.102 \mathrm{~m} / \mathrm{s}$. The behavior of all range ring intersection points and the points that are picked for the 2 DOF estimation are depicted in Fig. 5.

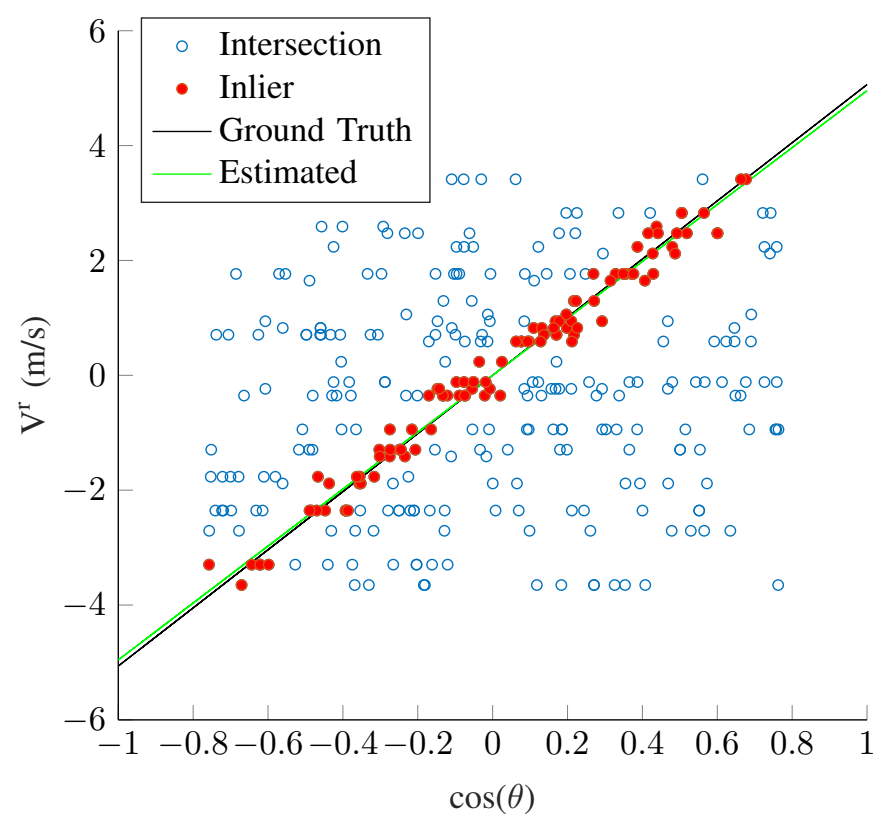

Fig. 5. Measurement results of the estimation in 2 DOF.

\section{CONCLUSION}

The proposed algorithm utilizes simple single-channel radar sensors to estimate the ego-motion of the car. The algorithm is able to extract valid points out of all range intersection points of a measurement. A setup with just 3 sensors and low velocity and range resolutions gives a good 2 DOF estimation of the movement. Future measurements will examine the 3 DOF estimation utilizing more sensors on both sides of the car to minimize the estimation error.

\section{REFERENCES}

[1] Shineonoat, "Free illustration of a hybrid car," 2017. [Online] Available: https://www.vecteezy.com/vector-art/113748-free-illustrationof-hybrid-car. [Accessed: 01- Nov- 2017].

[2] D. Kellner, "Verfahren zur Bestimmung von Objekt- und Eigenbewegung auf Basis der Dopplerinformation hochauflösender Radarsensoren," doctoral thesis, University of Ulm, 2016.

[3] Fischler, Martin A. and Bolles, Robert C. "Random Sample Consensus: A Paradigm for Model Fitting with Applications to Image Analysis and Automated Cartography," Communications of the ACM, vol. 24, no. 6, pp. 381-395, 1981.

[4] C. Croarkin, P. Tobias, and C. Zey, Engineering statistics handbook. NIST iTL, 2002. 